\title{
Towards Efficient P2P-based VoD Provisioning in Future Internet
}

\author{
Mostafa M. Fouda ${ }^{* 1}$, Tarik Taleb ${ }^{\ddagger 2}$, Mohsen Guizani ${ }^{\S 3}$, and Nei Kato ${ }^{\dagger 4}$. \\ *Faculty of Engineering at Shoubra, Benha University, Egypt. \\ ${ }^{\dagger}$ Graduate School of Information Sciences (GSIS), Tohoku University, Japan. \\ $\ddagger$ NEC Europe Ltd. \\ $\S$ Kuwait University, Kuwait. \\ Emails: mfouda@ieee.org ${ }^{1}$, tarik.taleb@nw.neclab.eu ${ }^{2}$, \\ mguizani@ieee.org ${ }^{3}$, and kato@it.ecei.tohoku.ac.jp ${ }^{4}$
}

\begin{abstract}
The current Internet framework is, no longer, able to support the heterogeneous networking technologies, mobile devices, increased number of users, and also the high user requirements for sophisticated services and applications. As a consequence, researchers envision the "future Internet" whereby all these issues may be effectively addressed. The future Internet architecture, amongst many components, is expected to comprise Peer-to-Peer (P2P) Video on Demand (VoD) streaming technologies. Since the conventional streaming techniques over $P 2 P$ frameworks have a number of shortcomings, design issues pertaining to the P2P VoD schemes need to be seriously reconsidered. In this paper, we envision a scheme to effectively provide a VoD using P2P-based mesh overlay networks that may be part of the future Internet. Our scheme also comprises domain-based localization and congestion awareness strategies for the selection of the most appropriate peers. Empirical results have demonstrated the effectiveness of this scheme in terms of scalability and capability to reduce the startup latency and total link cost, and to also ensure a sustainable playback rate that is crucial for providing VoD services over the future Internet.
\end{abstract}

Index Terms-Peer-to-Peer and Video-on-Demand streaming.

\section{INTRODUCTION}

In recent time, a plethora of services have evolved in order to meet the ever-increasing demands for information sharing. The current Internet architecture, however, was designed four decades ago, and is expected, in the near future, not to be able to satisfy the increasing users' requirements in terms of Quality of Service (QoS). Additionally, the Internet architecture is also missing crucial elements such as reliability, resiliency, security, mobility, context-awareness, and so forth. The two projects conducted by the National Science Foundation (NSF) in the USA called Global Environment for Network Innovations (GENI) [1] and Future InterNet Design (FIND) [2] aimed at addressing and identifying crosslayer design, network virtualization, dynamic switching of optical circuits, service discovery and composition, service management, traffic and routing engineering, and so forth. In Japan, the AKARI project, launched by the National Institute of Information and Communications Technology (NICT) [3], aims at innovating ideas, technologies, and a NeW Generation Network (NWGN) framework by 2015.

A major goal of the future Internet projects is to envision improved means of handling data traffic, substantial portion of which is currently generated by Peer-to-Peer (P2P) ap- plications employed for sharing files and streaming live/ondemand multi-media contents. These P2P applications involve a huge volume of information exchanged along both uplink and downlink directions amongst users/peers [4]. Compared to the more commonly used client/server-based applications evolved in the traditional Internet, P2P programs often select one or more suitable candidates from a pool of peers, which offer the same services or resources. This unique feature of P2P applications leads to increased reliability and resiliency to a single point of failure. The real-time video streaming or Video on-Demand (VoD) streaming techniques are expected to evolve further in the new business models that would make the future Internet sustainable. While live streaming systems are unable to provide user-interactive facilities (e.g., pause, random jumps, rewind, forward, slow motion, and so forth), the VoD systems can provide these convenient features to their subscribers. This issue makes the design and deployment of a $\mathrm{P} 2 \mathrm{P}-\mathrm{VoD}$ system in the future Internet rather difficult. Therefore, the research work on developing $\mathrm{P} 2 \mathrm{P}-\mathrm{VoD}$ architectures to fit the future Internet framework is a timely and important one. In order to effectively overcome this problem, we envision a localization-based P2P VoD scheme in this paper.

The rest of the paper is organized as follows. Section II presents an overview on some future Internet initiatives along with relevant research work on P2P VoD strategies. Section III also presents an overview on real time VoD P2P streaming systems. Our envisioned P2P-based VoD system is presented in Section IV. In Section V, we provide some empirical results to validate the performance of our envisioned system. Finally, the paper concludes in Section VI.

\section{RELATED WORK}

\section{A. Recent Future Internet Initiatives}

Among the ongoing research initiatives for dealing with the challenges of todays Internet and envisioning future Internet Networks, FIND [2] is a major project conducted by NSF. FIND focuses on the development of network architecture, security, advanced wireless and optical properties, economical principles, and in general, schemes to construct a global network in fifteen years from now. The GENI [1] program was initiated to address the lack of security, reliability, evolvability, and manageability of the current Internet. In Europe, the 
FIREworks (Future Internet Research and Experimentation Strategy Works) project [5] and the EIFFEL (Evolved Internet Future for European Leadership [6]) Support Action (SA) for the $7^{\text {th }}$ Framework Program (FP7) are notable examples of future Interet initiatives. While these projects have the same objective in broader terms, their methodology and design criteria are not necessarily similar. The conflict in design goals is also evident in the various Internet Engineering Task Forc (IETF) work groups. For instance, one IETF group envisioned Low Extra Delay BAckground Transport (LEDBAT) protocol [7], which attempts to minimize the additional latency invoked by the advanced networking applications expected in the future Internet. Another work group introduced the Diffserv (Differentiated services) Code Point for bulk traffic to identify, mark, and manage congestion events arising from P2P traffic under Diffserv framework. On the other hand, for managing the P2P traffic volume within an acceptable level at the network operators' expensive links, two further IETF groups were formulated, namely DECoupled Application Data Enroute (DECADE) [8] and Application Layer Traffic Optimization (ALTO) [9] working groups. The former identifies the problem with current Internet caches (e.g., P2P and web caches), which attempt to provide adequate storage capabilities within the network for reliable access of resources, but fail to explicitly support individual $\mathrm{P} 2 \mathrm{P}$ application protocols or user access to the content providers' caches. The latter intends to provide a simple mechanism to convey network information to the $\mathrm{P} 2 \mathrm{P}$ applications. This helps these $\mathrm{P} 2 \mathrm{P}$ applications to reduce the overhead associated with measuring topology information such as path performance metrics computation and so forth. In addition, the work in [10] proposed use of traffic localization for P2P applications by adding ALTO service to perform better than random initial peer selection.

\section{B. Related work on P2P VoD schemes}

To fit the future Internet, a number of research work has been devoted to combine P2P strategies with the serverclient streaming framework. A prime example is (BASS) [11], which consists of an external media server and a modified BitTorrent [12] protocol. The BASS clients are incapable of downloading contents prior to the current playback time. Rather, they receive chunks from the media server sequentially and do not require to obtain the already/being downloaded chunks by the BitTorrent. However, the shortcoming of the BASS framework consists in its lack of scalability when the system serves a large population of peers.

To deal with the afore-mentioned problem, no external media server was considered in the BitTorrent Streaming (BiToS) framework [13]. Instead of the "rarest-first" strategy, BiToS employs a selection mechanism, which ignores missing chunks (i.e., the chunks unable to meet their playback time). Since these missing chunks are not downloaded, they cannot be played back. This results in disrupted video playback.

The work in [14] focuses on providing small startup delays in $\mathrm{P} 2 \mathrm{P}-\mathrm{VoD}$ systems by integrating network coding with segment scheduling. Thus, it achieves a high utilization of the available resources. However, this approach does not consider practically deployable $\mathrm{P} 2 \mathrm{P} \mathrm{VoD}$ environments. In order to overcome this problem, it is important to design a scheduling mechanism capable of instructing the involved peers to assist one another in streaming the $\mathrm{VoD}$ contents in a real-time manner. To this end, the authors proposed a VoD scheme in an earlier work [15] over P2P mesh-based overlay networks with user-scalability features.

\section{OVERVIEW OF CONVENTIONAL P2P VOD STREAMING}

$\mathrm{P} 2 \mathrm{P}$-based streaming has recently emerged as an alternative to traditional server-based streaming (e.g., Youtube) for VoD provisioning. It already emerged as a promising technology for the future Internet targeting global users connected to heterogeneous networks. From the perspectives of a broadcaster/content provider, the $\mathrm{P} 2 \mathrm{P}$ approach provides an added incentive as it allows the servicing of a large audience (i.e., many peers) without the need for investment in additional resources. On the other hand, a user also experiences improved delivery rate of the multimedia content while he/she can also upload his/her own content to other peers. Most P2P VoD systems are either tree- or mesh-based. In the former, every node obtains data from a source/parent node according to a tree-like structure. In future Internet where such nodes are expected to frequently change, the tree needs to be constantly re-constructed. As a consequence, the children nodes fail to receive the streaming feed until the tree is re-constructed. On the other hand, in case of the mesh-based overlay network, a peer, which has just arrived at the overlay, contacts the tracker (e.g., a directory server) to obtain a list of a number of active peers. Having received the same, this new peer, at once, commences requesting the video chunks from the peers in the neighbor-list. It should also be noted that a peer is connected with a small subset of active peers at any given time, and it is allowed to exchange video chunks and control messages only with them. The overall architecture of the mesh-based $\mathrm{P} 2 \mathrm{P}$ VoD is depicted in Fig. 1. In contrast to its tree-based counterpart, mesh-based $\mathrm{P} 2 \mathrm{P}$ VoD networks are more resilient to node failures.

The conventional P2P-based VoD systems implicitly assume that a new user keeps watching the video stream until $(i)$ he/she leaves the mesh overlay, or (ii) the streaming session fails. This assumption naively excludes the user-interaction features (e.g., pausing, replaying, fast forwarding, and so on) in order to simplify the system design. However, in practice, instead of playing the video in a continuous fashion, the VoD subscribers tend to frequently jump to a more interesting section of the video either because they feel bored or do not have enough time to watch the entire video content. The frequent interactions of the users in the P2P VoD applications pose a serious challenge on the continuity of the video playback in terms of the "seeking delay", i.e., the time required since the request for a video segment till the segment becomes available. To have an almost zero seeking delay to watch the video without any interruption, each video segment needs to be prefetched by a peer prior to the playback of the segment. This 


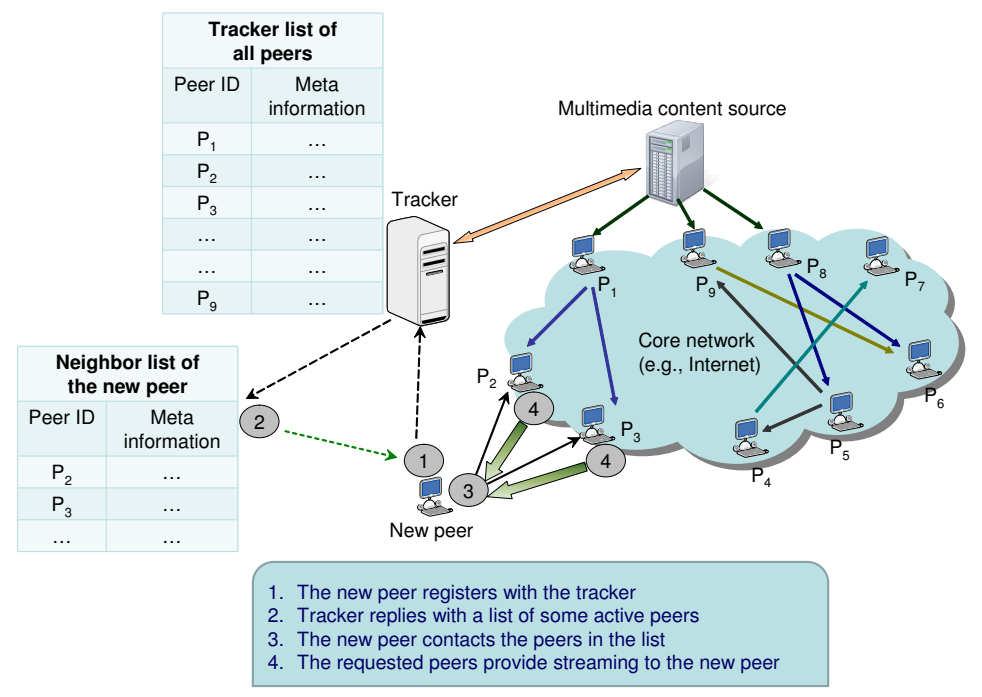

Fig. 1. The mechanism of a new host/peer joining the P2P-VoD overlay.

increases the playback continuity. However, the conventional $\mathrm{P} 2 \mathrm{P}$ VoD systems, based on their naive assumption that the users watch the video sequentially without any interactive "seek", perform the "pre-fetching" of video segments in a sequential manner. An alternative to this approach is to adopt a mechanism that pre-fetches random video segments. In the next section, we envision a pre-fetching mechanism for obtaining appropriate video segments $a$ priori at the peers in a mesh-P2P VoD environment.

\section{ENVISIONED P2P VoD SCHEME}

In the previous sections, we reasoned why it is important to design an effective P2P-VoD system that overcomes the shortcomings of its conventional counterparts, and meets the requirements of the future Internet architecture and the high expectations of its community of users. In this section, we envision a P2P VoD mechanism which utilizes the overlay network resources by considering the available upload bandwidth of the source node and that of each peer, and designing an efficient pre-fetching scheme coupled with a scheduling mechanism for obtaining a priori the appropriate video segments. It is worth noting that although our envisioned scheme provides a way to modify the existing P2P VoD designs on the current Internet, such enhancements are essential to deal with the new trends and design challenges of $\mathrm{P} 2 \mathrm{P}$ VoD in the future Internet.

Similar in spirit to the ALTO service [10], the envisioned system comprises a "tracker" containing the list of existing peers in the $\mathrm{P} 2 \mathrm{P}$ overlay along with their IP addresses, port numbers, upload capacities, and chunk bitmap information. Let $P_{s r c}, P_{r e c}$, and $P_{s r v}$ denote the source node (i.e., the content distributor), the receiving peer, and a selected peer to serve the receiving peer, respectively. When a new peer joins the considered P2P mesh overlay, it expects to receive video streaming from other nodes, and hence it is denoted by $P_{r e c}$. The tracker provides $P_{r e c}$ with a neighbor list, $N L$. Instead of arbitrarily selecting $P_{s r v}$, the envisioned scheme adopts the following steps in the tracker. First, the peers which contain the video segments following the last playable chunk of $P_{r e c}$ are chosen as potential $P_{s r v}$ candidates. Among these candidates, the tracker selects the ones with adequate upload capacity and the minimum appearance in the $N L$ s of the already receiving peers. This is done in order to distribute the load over the participating peers as per their available resources. Second, $P_{s r c}$ is not included in $N L$ in case the number of already chosen $P_{s r v}$ is sufficient. This is for the purpose of employing $P_{s r c}$ in only critical situations since it contains the entire video content. Third, if $N L$ of $P_{r e c}$ is incomplete, random peers may be chosen as $P_{s r v}$ and added to the $N L$.

From hereon, we describe the pre-fetching and scheduling algorithms, implemented in $P_{r e c}$ and $P_{s r v}$, respectively. The overall mechanism is shown in Fig. 2. The pre-fetching algorithm consists of two phases. In the first phase, $P_{\text {rec }}$ requests each $P_{s r v}$ in its $N L$ for specific bandwidth slots, where each bandwidth slot is sufficient for transferring a single video chunk. In order to enhance the utilization efficiency of the available bandwidth, the number of bandwidth slots requested by $P_{r e c}$ is set directly proportional to the upload capacity of each $P_{s r v}$, and should not exceed the maximum number of needed chunks available at the $P_{s r v}$. In addition, $P_{r e c}$ also sends its own Time-To-Freeze $(T T F)$, which is defined as the remaining time for the video playback to enter the freezing state. Fig. 3 depicts this through an example whereby $P_{\text {srv }}$ exploits the received $T T F$ value for prioritizing requests during the scheduling process, which is described next.

The scheduling phase of $P_{s r v}$ allocates its available upload bandwidth to the currently requesting $P_{\text {rec. }}$. $P_{s r v}$ assigns the highest priority to $P_{r e c}$ with the minimum TTF. The rational behind this is to ensure smooth playback (i.e., without freezing the video) at the concerned $P_{r e c}$. To achieve this, $P_{s r v}$ allocates up to $K \%$ of its available upload bandwidth to $P_{r e c}$ with a $T T F$ value less than that of $T T F_{t h}\left(T T F<T T F_{t h}\right)$, where $T T F_{t h}$ is a pre-defined threshold. The peer with the lowest TTF will be assigned the highest bandwidth. The hypothesis of the second prioritization lies on the observation 


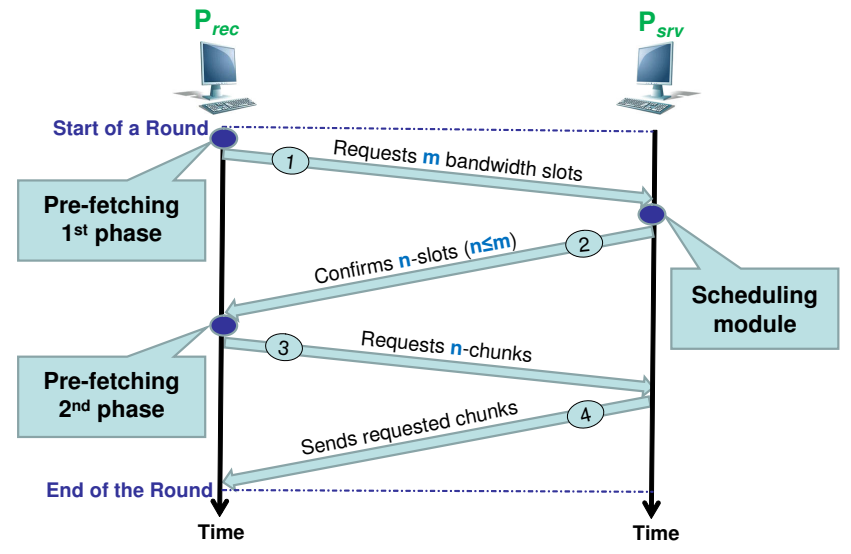

Fig. 2. Envisioned communication algorithm between $P_{r e c}$ and $P_{s r v}$ comprising pre-fetching and scheduling.

that the currently requesting $P_{r e c}$ with higher upload capacities should be assigned more bandwidth by $P_{s r v}$ so that those $P_{r e c}$ may obtain more chunks to utilize their upload capacities efficiently while serving contents to other peers. It is worth mentioning that the $T T F$ of a newly arriving peer that has not yet received any chunk is zero; a fact that renders the priority of the new peers in question to be the highest. This decreases the startup latency of the $P_{\text {rec }}$ 's playback to a minimum level. Following the scheduling process, $P_{s r v}$ sends a specific number of bandwidth slots to each requesting $P_{r e c}$ that initiates the second phase of pre-fetching. In this second phase of pre-fetching, $P_{r e c}$ determines the appropriate video chunks, which are to be requested from $P_{s r v}$.

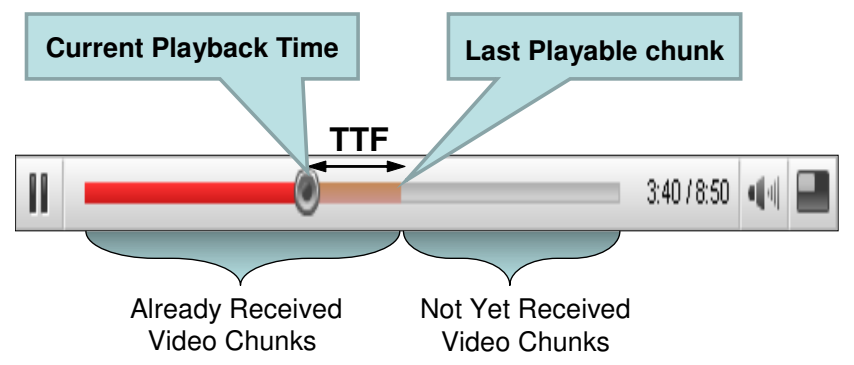

Fig. 3. Depiction of Time-To-Freeze $(T T F)$ parameter in a typical example.

Since the main objective of the envisioned system is to maintain smooth playback, the priority in requesting video chunks is given towards those particular chunks, which appear just after the playback time with a maximum interval equal to a pre-defined time window, $T$. After having all chunks within $T$ being requested, $P_{r e c}$ considers that the streaming quality will not degrade during the next $T$ time window. After this, there is no need to request chunks sequentially. On the contrary, at this point, if $P_{r e c}$ can ask for more chunks from its corresponding $P_{s r v}$, it may consider requesting chunks randomly to increase the chunks diversity in the overlay.

Next, we focus on peer selection ability of the tracker. In the envisioned P2P VoD scheme, the tracker is assumed to be aware of the network topology, i.e., the different domains of the network. It is also assumed to acquire functionalities for assessing network link congestion over the different network domains (e.g., through deployment of monitoring agents). The domain-based localization refers to the selection of "nearby" peers belonging to the same domain. As selecting a nearby peer is not always an optimal decision (e.g., in case a segment of the link to a nearby peer is congested), link congestion should be also considered in the selection of peers. The two cases are depicted in a simple scenario in Fig. 4. There are four domains in this scenario, namely $D_{1}, D_{2}, D_{3}$, and $D_{4}$. Let us consider peers $P_{1}, P_{2}$, and $P_{3}$ belonging to domain $D_{1}$. Let $d_{P_{i}, P_{j}}$ refer to the end-to-end propagation delay between two neighboring peers $P_{i}$ and $P_{j}$. In this example, we consider $d_{P_{1}, P_{2}}<d_{P_{1}, P_{3}}$. While searching for its neighboring peers, $P_{1}$ may be assigned to either $P_{2}$ or $P_{3} . P_{2}$ and/or $P_{3}$ may be chosen according to the domain-based localization scheme. However, when there is congestion in the link between $P_{1}$ and $P_{2}$, the congestion awareness scheme selects $P_{3}$ based on the network domain and the link congestion.

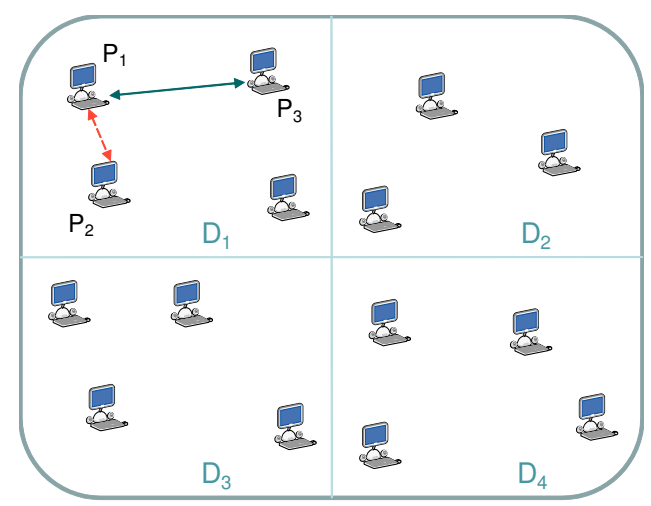

Fig. 4. Domain-based localization vs. congestion awareness strategies for peer selection.

\section{Performance Evaluation}

We conducted simulations using Matlab [16] to verify the effectiveness of the proposed selection strategies: domainbased localization and congestion awareness. For the simulation topology, we considered four different domains. A single peer is considered to be the server at the beginning. The serviced video file size is set to $16 \mathrm{Mb}$. The chunk size is set to $8 \mathrm{~Kb}$. We considered six neighboring peers. The tracker update period is set to $5 \mathrm{~s}$. A population of 200 peers is used. The peers belong to four peer classes, namely A, B, C, and D with different upload and download bandwidths as used in our former approach [15]. These four peer classes are employed in order to address heterogeneity due to the different bandwidth of the access network.

Two performance metrics are considered for evaluating the envisioned approach, namely the maximum supportable playback rate and total link cost. Given the startup delay (or initial buffering), the maximum supportable playback rate is defined as the maximum rate at which the video can be played without being frozen. On the other hand, the total link cost refers to the cost of transferring chunks to all the 


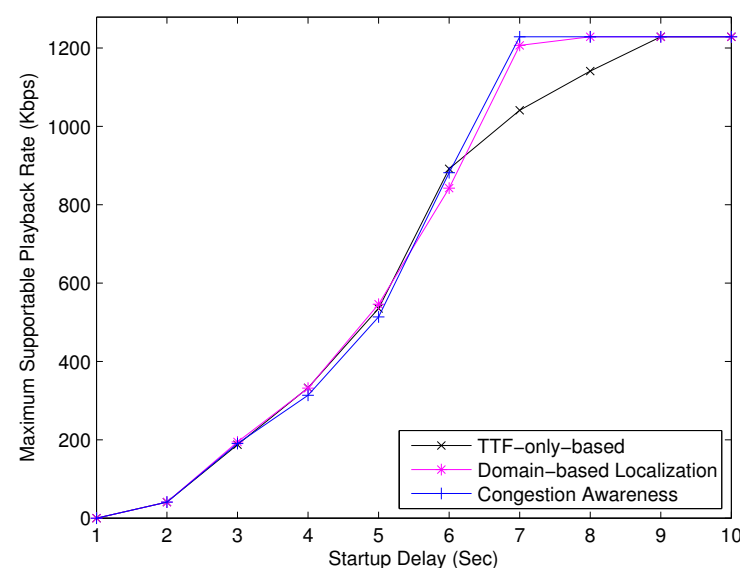

Fig. 5. The maximum supportable playback rates for different startup delays.



Fig. 6. Total link cost in the considered schemes.

receiving peers for a particular VoD stream. The total link cost is represented by a weight depending on the congestion of the link, and the distance between the serving and the receiving peers.

We compare the performance of our former scheme, TTFonly-based [15] against the proposed enhancements in terms of domain-based peer localization and congestion-aware peer selection. We envision a flash crowd scenario, which represents the worst case scenario of a VoD streaming system over $\mathrm{P} 2 \mathrm{P}$ overlay. The comparison results are presented in Figs. 5 and 6. In Fig. 5, the maximum supportable playback rate is plotted for the considered schemes over different startup delays. As evident from this result, both the domainbased peer localization and congestion-aware peer selection schemes outperform the original one. With the two proposed enhancements, the peers can watch the video stream at the maximum supportable playback rate when the startup delay is approximately $7 \mathrm{~s}$. Our previous scheme achieves the maximum playback rate at a later time. This indicates that the user can enjoy smooth playback at an earlier time instance with the proposed enhancements. On the other hand, Fig. 6 depicts the total link cost associated with the considered schemes. Our previous VoD scheme contributed to the highest total link cost, i.e., 4.2 units. This is because of the fact that the far-away peers may be chosen as neighbors in the previous scheme. The domain-based localization peer selection strategy overcomes this issue as evident by its lower cost (approximately 3 units). The congestion-aware mechanism improves the performance further by selecting the less congested peers.

\section{CONCLUSION}

In this paper, we pointed out the need for effective streaming of P2P VoD multimedia contents over the future Internet. To ensure quality of experience for subscribers of such a $\mathrm{P} 2 \mathrm{P}$ VoD service, it is essential to ensure short startup delay, smooth video playback, and scalability. These conditions may be highly affected by the upload bandwidth utilization of the involved peers. The success of a P2P VoD system in the future Internet hinges on three key elements, namely an effective pre-fetching mechanism for obtaining the necessary video segments, an efficient scheduling algorithm for allocating the upload bandwidth, and an appropriate peer selection strategy for selecting the best possible peers. In line with these, we proposed a P2P VoD pre-fetching and scheduling mechanism and integrated with it domain-based peer localization and congestion-aware peer selection schemes. We validated the performance of the proposed scheme using computer simulations. The proposed mechanism exhibits encouraging performance even when the system is operating under worst case scenarios, servicing a potential flash crowd of users.

\section{ACKNOWLEDGMENT}

This work is partially supported by the Government of Egypt.

\section{REFERENCES}

[1] J. B. Evans and D. E. Ackers, "Overview of GENI and Future Internet in the US," May 22, 2007; http://www.geni.net/

[2] NSF NeTS FIND Initiative; http://www.nets-find.net/

[3] T. Aoyama, "A New Generation Network: Beyond the Internet and NGN," IEEE Communications Magazine, vol. 47, no. 5, May 2009, pp. 82-87.

[4] http://torrentfreak.com/cisco-expects-p2p-traffic-to-double-by-2014$100611 /$

[5] FIREworks; http://www.ict-fireworks.eu/

[6] EIFFEL; http://www.fp7-eiffel.eu/

[7] S. Shalunov, M. Sridhavan et al., "LEDBAT Working Group Charter," http://www.ietf.org/dyn/wg/charter/ledbat-charter.html

[8] S. Haibin, R. Woundy et al., "DECADE Working Group Charter," http://trac.tools.ietf.org/bof/trac/wiki/decade

[9] J. Peterson, E. Marocco, V. Gurbani et al., "ALTO Working Group Charter," http://www.ietf.org/html.charters/alto-charter.html

[10] J. Seedorf, S. Kiesel, and M. Stiemerling, "Traffic Localization for P2PApplications: The ALTO Approach", in Proc. IEEE P2P'09, Seattle, WA, USA, Sep. 2009.

[11] C. Dana, D. Li, D. Harrison, and C. Chuah, "BASS: BitTorrent assisted streaming system for video-on-demand," in Proc. International Workshop on Multimedia Signal Processing (MMSP), Shanghai, China, Nov. 2005.

[12] "BitTorrent," http://www.bittorent.com.

[13] A. Vlavianos, M. Iliofotou, and M. Faloutsos, "BiToS: Enhancing BitTorrent for supporting streaming applications," in Proc. IEEE Global Internet, Barcelona, Spain, Apr. 2006.

[14] S. Annapureddy, S. Guha, C. Gkantsidis, D. Gunawardena, and P. Rodriguez, "Exploring VoD in P2P Swarming Systems," Proc. IEEE INFOCOM'07, Alaska, USA, May 2007.

[15] M. Fouda, T. Taleb, M. Guizani, Y. Nemoto, and N. Kato, "On Supporting P2P-based VoD Services over Mesh Overlay Networks," Proc. IEEE GLOBECOM'09, Hawaii, USA, Dec. 2009.

[16] "Matlab," http://www.mathworks.com/ 